\title{
A general approach for calculating the likelihood of dyadic interactions: Applications to sex preferences in rat pups and agonistic interactions in adults
}

\author{
JEFFREY C. SCHANK \\ University of Califormia, Davis, California \\ and \\ JEFFREY R. ALBERTS \\ Indiana University, Bloomington, Indiana
}

\begin{abstract}
We introduce a general approach for calculating the expected frequency of different types of dyadic interactions between animals and illustrate it with two applications. The first application determines whether there are sex contact biases between male and female Norway rat pups. We hypothesized that opposite-sex contact biases should be small, because it is likely that differences in contact based on the sex of animals would interfere with other biological functions, such as group temperature regulation. We found a small but significant bias of $3 \%$ in opposite-sex contacts. The second application reanalyzed J. B. Calhoun's (1962) data on antagonistic encounters between rats for two factors (sex and birth location), each with two levels. The results of these two applications suggest that sex contact biases among pups are predictive of agonistic encounters among adults and that this approach has general application to the analysis of dyadic interactions among animals.
\end{abstract}

An important, nearly ubiquitous phenomenon in animal behavior is dyadic interactions (e.g., contact) between two animals (e.g., male and female). Among Norway rats, for example, contact is so prominent that Barnett (1963) has described Rattus norvegicus as a "contact species." Despite the ubiquity of dyadic interactions between animals, we have no general or systematic approach for calculating their expected frequencies. Thus, the primary aim of this paper is to present a general framework for calculating the likelihood of dyadic interactions between different types of individuals.

We also have a specific experimental aim that motivated the development of this approach-namely, to reexamine the problem of whether there is an opposite-sex contact bias among rat pups 10 days of age and younger. Deni, Martin, Makin, Longo, and Andres (1981) reported that rat pups, 5-9 days of age, exhibited sex preferences, as measured by physical contact between individual pups in the context of a huddle. Subsequently, Stollnitz (1982) pointed out that it was likely that the apparent sex preferences reported by Deni et al. were due to statistical bi-

This work was supported by the NSF through the Center for the Integrative Study of Animal Behavior (CISAB) and by the NIH (NIH 1 F32 HD088188-01 to J.C.S. and NIH MH 28355 to J.R.A.). We also thank the referees of this paper for clarifying and correcting some points made in the paper. Correspondence concerning this article should be addressed to J. C. Schank, Department of Psychology, University of California, One Shields Ave., Davis, CA 95616 (e-mail: jcschank@ucdavis.edu). ases in the number of contacts that can form between males and females in a huddle and not to sex preferences. That is, if there are three females and three males in a huddle, an individual can contact only two individuals of the same sex but three individuals of the opposite sex. This suggests that the distribution of pup contacts should be in the ratio of same-sex to opposite-sex contacts, which is $2: 3$ (Stollnitz, 1982) and not $1: 1$, as was tacitly assumed by Deni et al. Our general approach for calculating the likelihood of dyadic interactions allows us to reexamine this problem with greater theoretical and methodological precision.

Our precept is to treat a group of animals as a network in which individuals are nodes and interactions between nodes, such as contact, are represented as connections between nodes. With this conceptualization, calculating the expected frequency distribution of dyadic interactions between different types, categories, or classes of animals is straightforward. In addition to describing how expected frequencies of dyadic relationships can be calculated with this approach, we will describe a computer application and algorithm for collecting contact data that utilizes this "network" perspective and nearly eliminates experimenter error in tracking rats. We then show that this approach can be generalized to a hierarchy of types (analogous to factors and levels in an analysis of variance) by calculating the appropriate probabilities and conditional probabilities for different dyadic-agonistic interactions between the adult rats presented in Calhoun (1962). 


\section{APPLICATION I: Calculating the Frequencies of Sex Contact Among Pups}

Deni et al. (1981) reported that Norway rat pups, 59 days of age, exhibited opposite-sex preferences, as indicated by physical contact between individual pups in a huddle. Subsequently, Stollnitz (1982) pointed out that it was likely that the apparent sex preferences reported by Deni et al. were due to statistical biases in the number of contacts that can form between males and females in a huddle. When we reexamined the results reported by Deni et al. and calculated the expected frequency distribution, we did not find significant deviations from the expected frequencies of same- and opposite-sex contact (see the $95 \%$ confidence intervals calculated in Table 1 ). However, the data do suggest that there may be a small opposite-sex contact bias, perhaps in the range of $2 \%-5 \%$ (Table 1). Without an appropriate analysis, it is still an open question as to whether there are contact differences between male and female pups.

Based on the sensorimotor limitations of young pups (Alberts, 1978b) and the physiological function of huddling by pups (Alberts, 1978a), it seems reasonable to predict that opposite-sex contact differences must be either very small or nonexistent. Large differences in sexrelated contact would present a paradox: Group regulatory behavior serves energy conservation and body temperature maintenance, and so large contact differences would surely be maladaptive. There is no reason to think that sex-related contact differences coincide with temperatureand energy-related contact. Indeed, they cannot coincide, because the sex of a pup is constant, whereas temperaturerelated contact among pups varies as a function of ambient temperature and the internal state of each pup (Alberts, 1978a). Thus, it is likely that the early developmental emergence of large sex differences in contact would conflict with the behavioral interactions required for temperature regulation and energy conservation.

If there are opposite-sex preferences in pup aggregation, it is likely that they are expressed as small deviations from the expected frequency of contact between the sexes. Very precise methods are required to detect such differences, if in fact they exist. To solve this problem, we have developed a method for calculating expected frequencies of dyadic contacts. If pups do show sex preferences early in development, this approach should allow the detection of very subtle sex-related differences in contact.

\section{Method}

\section{Animals}

The animals consisted of 19 litters of 8 rat pups (Sprague-Dawley, originally obtained from Taconic Farms, Germantown, NY), 10 days old, born at Indiana University. At 3 days of age (day of birth = Day 0 ), litters were culled to 4 females and 4 males. Litters and dams were housed in standard polypropylene maternity cages $(48 \times 20$ $\times 26 \mathrm{~cm}$ ). Colony rooms were maintained at $24^{\circ} \pm 2^{\circ}$ and illuminated from 0800 to $2000 \mathrm{~h}$. The methods used were observational and entirely noninvasive.

\section{Procedure}

Contact among 8 rat pups in the nest can be difficult to accurately and reliably observe. In particular, the three-dimensional geometry of a clump of pups can hide or even create contact biases. The latter possibility is a consequence of the frequency dependence of contact. If, for example, opposite-sex contact tended to be slightly more stable, such contacts might be obscured by other pups climbing on top of the huddle. Counting the actually observed types of contact may create a bias toward detecting more same-sex contacts and fewer opposite-sex contacts, thereby creating a bias opposite to what actually occurred.

We have found, however, that on a flat and level surface, contact among pups is easily observed and pups freely move about initiating and maintaining contact with other pups (Schank \& Alberts, 1997). Only rarely do they crawl over or under each other (unpublished observations).

Aggregation among pups and other objects is mediated by thermal, tactile, and olfactory modalities (Alberts, 1978b; Alberts \& Brunjes, 1978). The lability of an infant pup's thermal and metabolic status (e.g., Alberts, 1978b) creates variable responses to temperature cues. To control for thermal effects, aggregative behavior occurred in a $30 \times 20 \times 5 \mathrm{~cm}$ aluminum temperature-controlled $\left(34^{\circ} \mathrm{C} \pm 0.2^{\circ} \mathrm{C}\right.$ ) arena (Schank \& Alberts, 1997). A rack was used to randomly arrange pups in an evenly spaced distribution across the surface of the arena at the start of an experimental session. The rack was constructed of $7.62 \times .95 \mathrm{~cm}$ laths of balsa wood painted white, creating a frame with two identical rows of eight stalls, 4.125 $\times 5.625 \mathrm{~cm}$, for holding individual pups.

At the start of an experimental session, the pups were placed individually in each of the eight stalls within the rack. Lifting the rack simultaneously and unobtrusively released the eight pups to move

Table 1

Data on Same- and Opposite-Sex Contacts Reported by Deni, Martin, Makin, Longo, and Andres (1981), Together With the Calculated Expected Frequencies and the $95 \%$ Confidence Intervals for Their Data

\begin{tabular}{llrrrrr}
\hline Day & Contact & Mean & Frequency & Expected & Difference & Confidence Interval \\
\hline 6 & same & 6.1 & .374 & .4 & -.026 & .23 \\
& opposite & 10.2 & .626 & .6 & .026 & .30 \\
7 & same & 7.9 & .346 & .4 & -.054 & .11 \\
& opposite & 14.9 & .654 & .6 & .054 & .14 \\
8 & same & 8.1 & .401 & .4 & .001 & .14 \\
& opposite & 12.1 & .599 & .6 & -.001 & .14 \\
9 & same & 7.3 & .397 & .4 & -.003 & .20 \\
& opposite & 11.1 & .603 & .6 & .003 & .19 \\
\multirow{2}{*}{ Total } & & 7.35 & .378 & .4 & -.022 & .08 \\
& & 12.08 & .622 & .6 & .022 & .11 \\
\hline
\end{tabular}


around the arena. Before each session, the arena was cleaned with ethyl alcohol to minimize the effects of odors left by previous litters. Experiments were recorded by time-lapse video (12:1) with a camera placed directly above the arena. Videotapes were analyzed using NIH Image 1.60 for the Power Macintosh, as will be described below.

\section{Data Collection}

Observing the interactions among 8 pups, even on a flat surface, remains a challenging task. There is ample opportunity to err in assigning the appropriate sex to the individuals in contact. If no errors are made, it is a mathematical fact that the standard deviations of the frequency of the same-sex and opposite-sex contacts must be the same (i.e., because the frequency of opposite-sex contacts is one minus the frequency of same-sex contacts).

Deni et al. (1981) reported $81 \%$ interobserver reliability in encoding contact behavior among rat pups in their videotaped experiment. They also reported the standard deviation for the number of same-sex contacts as 3.8 and that for opposite-sex contacts as 4.9. This difference in standard deviations is within the bounds of experimenter error in correctly assigning sex to individuals in contact. Such experimenter error can be eliminated by a computer algorithm that keeps track of the sex of individuals.

Using a version of NIH Image modified to record and save data in spreadsheet form, a macro was written for scoring and recording contacts between individuals at 5-sec intervals. The underlying algorithm and strategy for entering pairs of animals in contact can be outlined succinctly. (1) A stack of images is created by taking an image from a 15-min clip of video every $5 \mathrm{sec}$, for a total of $180 \mathrm{im}$ ages per litter. (2) At the start of a scoring session, the observer is prompted for the sex of each pup. (3) For each image thereafter, the observer enters the dyadic relationships formed among the pups in contact (e.g., "12 1324252834376778 " indicates nine dyadic relationships between pups in contact). Errors in entering dyads can occur without a rule for entering them. The rule used here was to start with Pup 1 and list all the pups it contacts, then go to Pup 2 and list all the pups it contacts that are greater than 2 , and so on until all such dyads are listed. This avoids listing both " 12 " and " 21 ." (4) The algorithm then looks up the sex of the animals for each pair of numbers and classifies the pair as either male-male, female-male, or female-female.

Once the sex is known for each pup at the start of an experimental session, this algorithm eliminates further errors in assigning sex to animals forming dyads; thus, the standard deviations for the data on same-sex versus opposite-sex dyads are always the same. It does not, however, eliminate two other possible sources of error. First, mistakes can be made in determining the sex of a pup prior to the start of an experimental session. In practice this almost never occurs, especially for 10-day-old rats. Second, it is possible to misidentify a pair of animals in contact during an experimental session. This kind of error, however, is independent of the sex of the pups and, thus, should have no statistical influence on the outcome. Indeed, no such errors were found for the 19 litters reported here upon rechecking the data for this type of error.

\section{Frequency of Dyadic Interactions}

Assumptions. In a group of $n$ individuals there are $r$ types of individuals (e.g., $r=2$ for male and female), and each type consists of $n_{i}$ individuals ( $i=$ the type of individual; e.g., $i=\mathrm{m}$, f for males and females). Thus, for males and females there are $n_{\mathrm{m}}$ males and $n_{\mathrm{f}}$ females. For males and females, there are three types of dyad: male-male $(m, m)$, female-male $(f, m)$, and female-female ( $f, f)$. (We shall always refer to opposite-sex dyads as female-male dyads, but this is also equivalent to male-female dyads.) Self-contact or self-association is excluded from this analysis.

Counting dyads. If the only types of individual in a group are male and female, there are $r=2$ types of individual and 3 types of dyad. In general, the number of types of dyad in a group is $r(r+1) / 2$.
In order to calculate the expected frequency of each dyadic type in a group, we must count the total number of possible types and the number of possible dyads for each type of dyad.

Counting dyads is easily accomplished by counting ordered dyads. For example, if there are three individuals, there are six ordered dyads: $(1,2),(2,1),(1,3),(3,1),(2,3)$, and $(3,2)$. The number of unordered dyads is always one half the number of ordered dyads. There are situations in which counting ordered interactions may be important, such as that in which there may be reciprocal grooming between a pair of animals. In many other cases, the order of the interactions is not important, such as that with pup contact and agonistic interactions between adult rats, as will be discussed in the next section.

The total number of unordered dyads (in the remainder of this paper, when we refer to dyads, we will be referring to unordered dyads), $K_{\mathrm{T}}$, is given by

$$
K_{\mathrm{T}}=\frac{n !}{2(n-2) !}=\frac{n(n-1)}{2},
$$

where $n$ is the total number of individuals in a group. For interactions between the same type of individuals, we have

$$
K_{i i}=\frac{n_{i}\left(n_{i}-1\right)}{2},
$$

and for interactions between different types of individuals, we have

$$
K_{i j}=n_{i} n_{j}
$$

We can predict the ratio of same-sex to opposite-sex contacts as a $2 K_{i i}: K_{i j}$. Using Equations 2 and 3, for $r=2$ and $n_{i}=n_{j}$, this ratio is $n_{i}\left(n_{i}-1\right): n_{i} n_{j}$, which is equivalent to the ratio $\left(n_{i}-1\right): n_{j}$. If $n=6$ and $n_{j}=n_{j}=3$, we get a ratio of $2: 3$, as was predicted by Stollnitz (1982).

The expected frequency distribution for different types of dyad in a group is obtained by dividing Equations 2 and 3 by Equation 1:

$$
P[(i, i)]=\frac{K_{i i}}{K_{\mathrm{T}}}=\frac{n_{i}\left(n_{i}-1\right)}{n(n-1)} \text { and } P[(i, j)]=\frac{K_{i j}}{K_{\mathrm{T}}}=\frac{2 n_{i} n_{j}}{n(n-1)} \text {. }
$$

For example, for $n=6$ and $n_{i}=n_{j}=3$, the frequency distribution of male-male, female-male, and female-female dyads is $0.2,0.6$, and 0.2 , respectively.

Statistical method. Whether in a huddle in the nest or aggregated on the surface of the arena, not all individuals will be in contact with all the other individuals in the group, and the connectivity of the group will change with time. Indeed, for there to be deviations from the expected frequency of a dyadic type, the connectivity of the group must be less than maximum. As long as there are interactions between individuals, however, the expected frequency of each type of dyad remains the same. The observed frequency of each type is obtained by taking the observed (obs) number of occurrences of the dyadic type and dividing it by the observed total number of dyads:

$$
\begin{aligned}
P_{\mathrm{o}}[(\mathrm{m}, \mathrm{m})] & =\frac{\mathrm{obs}\left(K_{\mathrm{mm}}\right)}{\mathrm{obs}\left(K_{\mathrm{T}}\right)}, P_{\mathrm{o}}[(\mathrm{f}, \mathrm{m})]=\frac{\mathrm{obs}\left(K_{\mathrm{fm}}\right)}{\mathrm{obs}\left(K_{\mathrm{T}}\right)}, \\
P_{\mathrm{o}}[(\mathrm{f}, \mathrm{f})] & =\frac{\mathrm{obs}\left(K_{\mathrm{ff}}\right)}{\mathrm{obs}\left(K_{\mathrm{T}}\right)} .
\end{aligned}
$$

For example, for Litter 17 (see Table 2), 1,966 dyads were observed in 180 observations of the litter and 394 female-female dyads were observed. Using Equation 5, this yields an observed frequency of $394 / 1,966=.2004$. Since the observed frequency distribution of dyadic types was based on a large number of contacts per litter, we expected that the data for the 19 litters would have low variance, making it possible to detect small deviations from the expected frequencies of dyadic types. 
Table 2

Frequency of Opposite-Sex, Female-Female, and Male-Male Contacts for 19 Litters

\begin{tabular}{cccc}
\hline \multicolumn{1}{c}{ Litter } & Opposite-Sex & Female- Female & Male-Male \\
\hline 1 & .6586 & .1908 & .1506 \\
2 & .5857 & .2318 & .1825 \\
3 & .6281 & .1221 & .2497 \\
4 & .6454 & .1252 & .2294 \\
5 & .6060 & .1885 & .2055 \\
6 & .6771 & .1553 & .1676 \\
7 & .6009 & .2099 & .1892 \\
8 & .5571 & .2411 & .2018 \\
9 & .5436 & .2256 & .2308 \\
10 & .6505 & .1903 & .1591 \\
11 & .4772 & .2807 & .2421 \\
12 & .6885 & .2184 & .0931 \\
13 & .5584 & .2703 & .1713 \\
14 & .5493 & .2155 & .2352 \\
15 & .6748 & .1988 & .1264 \\
16 & .6027 & .2389 & .1584 \\
17 & .6012 & .2004 & .1984 \\
18 & .5438 & .2065 & .2498 \\
19 & .6625 & .2145 & .1230 \\
$\bar{P}_{\mathrm{o}}[(i, j)]$ & .6059 & .2066 & .1876 \\
$P[(i, j)]$ & .5714 & .2143 & .2143 \\
$\left|\bar{P}_{\mathrm{o}}[(i, j)]-P[(i, j)]\right|$ & .0344 & .0077 & .0267 \\
$S_{D}$ & .0574 & .0411 & .0458 \\
$d f$ & 18 & 18 & 18 \\
$t$ & 2.6154 & 0.8189 & 2.5412 \\
Significance & $p \leq .0088 *$ & $p \geq .4235 \dagger$ & $p \leq .0205 \dagger$ \\
Confidence Interval & .0278 & .0199 & .0222 \\
\hline$*$ & & &
\end{tabular}

*one-tail $t$ test. 'two-tail $t$ test.

The observed frequencies of different dyadic types are not independent. Thus, each dyadic type (e.g., female-male) should be tested independently of the other dyadic-type frequencies in a parametric test. Deviations from the expected frequency of dyads for each type can be tested using the $t$ statistic by substituting Equations 4 and the mean, $\bar{P}_{o}((i, j))$, of the observed frequencies defined by Equation 5 into the one-sample $t$ test formula

$$
t=\frac{\left|P_{\mathrm{o}}[(i, j)]-P[(i, j)]\right|}{s_{D}} \sqrt{n},
$$

for $n$ litters having standard deviation $s_{D}, n-1$ degrees of freedom, where $i, j=\mathrm{f}, \mathrm{m}$ for opposite-sex contacts and $i, i=\mathrm{m}, \mathrm{m}$ or $\mathrm{f}, \mathrm{f}$ for same-sex contacts. A one-tailed $t$ test will be used to test the hypothesis that there were opposite-sex preferences expressed early in development, as was previously hypothesized by Deni et al. (1981), with $\alpha=.05$. Two additional comparisons will be made for male-male and female-female contacts. Because we did not hypothesize a direction for these contacts, both $t$ tests will be two-tailed, and to reduce the possibility of a Type I error, the significance level is halved, $\alpha=0.025$, for these tests.

\section{Results}

Ten-day-old rat pups exhibited sex differences in contact that deviated from the expected frequency by about $3 \%$ $(p<.0088$, one-tailed; see Table 2$)$. Further analysis revealed that male-male contacts were significantly less than expected ( $p<.0205$, two-tailed), whereas female- female contacts were not $(p>.4235$, two-tailed, see Table 2$)$.

\section{Discussion}

Small but significant sex differences were found in contact among 10-day-old Norway rat pups. Opposite- sex contacts were significantly higher than expected, male-male contacts were significantly less than expected, and female-female contacts were consistent with chance interactions. However, our methods were not designed to identify the source of the differences in contact (i.e., the sex preferences of male and female pups). The reduced contact seen in the frequency of male-male pairs suggests that focusing on opposite-sex contact alone may not reveal the sexual preferences that form early in development. For example, rather than opposite-sex preferences emerging by Day 10 , perhaps we observed male-male avoidance or some combination of male-male avoidance and opposite-sex preference. Clearly, more research is required to determine how sex preferences may have produced these patterns of contact.

\section{APPLICATION II: Calculating Frequencies of Agonistic Encounters}

Our method for calculating the frequency of dyads in a group of individuals is broadly applicable to other species and other social situations. To better illustrate the generality of this method and the errors it avoids, we have reanalyzed a study by Calhoun (1962). Over a period of 27 months, he observed various aspects of the behavior of wild rats living in an outdoor pen $(100 \times 100 \mathrm{ft})$ designed to mimic features found in the local urban ecology. Calhoun (1962, pp. 196-197) described his investigation of how the locality of birth of the wild rats in his pen affected their subsequent social status in the colony. His measures were the number of agonistic encounters between pairs of rats and which rat won the encounter. Each rat was either male or female and was born in either the southeast or the northwest half of the pen. Thus, there were two factors, sex (male and female) and birth locality (southeast and northwest). A rat could, for example, be a female born in the northwest half of the pen, or it could be a male born in the southeast half of the pen.

Calhoun (1962) assumed that "if $a$ denotes the number of southeast males and $b$ the number of northwest males, the interactions would be proportional to $a^{2}$ (southeast with southeast) $+2 a b$ (southeast with northwest) $[+] b^{2}$ (northwest with northwest).... The expected femalefemale and female-male encounters were similarly derived" (p. 197). This assumed that the appropriate null model for agonistic encounters is to randomly draw two individuals from either population with replacement. However, this is not quite the correct random model, since individuals cannot have agonistic encounters with themselves. If we remove self-agonistic interactions, the terms $a^{2}$ and $b^{2}$ are replaced by $a(a-1)$ and $b(b-1)$, respectively.

Let $\mathrm{m}$ denote male, $\mathrm{f}$ denote female, se denote the southeast half of the pen, and nw denote the northwest half. Agonistic encounters are represented by dyads of the four different types, $r=4$. For example, an interaction between a male from the northwest half and a female from the southeast half would be represented by (mnw, fse). Since the number of types of dyad is given by $r(r+1) / 2$, 
there are 10 types of dyadic interactions. The numbers of males and females from each birth location were $n_{\text {mse }}=$ $51, n_{\mathrm{fse}}=34, n_{\mathrm{mnw}}=25$, and $n_{\mathrm{fnw}}=27$. From these numbers, we can calculate the numbers of males $\left(n_{\mathrm{m}}=n_{\mathrm{mse}}+\right.$ $\left.n_{\text {mnw }}=76\right)$, females $\left(n_{\mathrm{f}}=n_{\text {fse }}+n_{\text {fnw }}=61\right)$, southeastside rats $\left(n_{\mathrm{se}}=n_{\mathrm{fse}}+n_{\mathrm{mse}}=85\right)$, and northwest-side rats $\left(n_{\mathrm{nw}}=n_{\mathrm{fnw}}+n_{\mathrm{mnw}}=52\right)$ and the total number of rats $(n=137)$.

Calhoun (1962) used the $\chi^{2}$ statistic to determine whether there was a significant difference in the numbers of winners and losers from the southeast and northeast halves of the pen. By using the approach developed here, however, we can calculate expected numbers and thereby test all possible hypotheses, using the $\chi^{2}$ statistic without the error of tacitly assuming self-agonistic encounters. (This assumption leads to large error only with small sample sizes; this is why our analysis does not qualitatively alter Calhoun's original analysis.)

Table 3 illustrates several cases. First, to determine whether there are differences in the frequency of agonistic encounters between male-male, male-female, and female-female dyads, we must calculate the probabilities of male-female encounters - an analysis Calhoun (1962) did not carry out. For male-male encounters, the appropriate probability is given by Equation 4:

$$
P[(\mathrm{~m}, \mathrm{~m})]=\frac{n_{\mathrm{m}}\left(n_{\mathrm{m}}-1\right)}{n(n-1)} .
$$

The probabilities for female-male and female-female are similarly defined (Table 3 ). By calculating these probabilities and using the $\chi^{2}$ statistic, we see that the frequency of agonistic encounters for male-male pairs is much greater than chance, for female-male pairs is much less than chance, and for female-female pairs is very close to chance (Table 3 ).

Conditional probabilities also can be defined for nested types of dyadic encounters. For example, the probability of female-male encounters from the two birth localities are conditionalized on the presence of an encounter between a female and male ( $f, m)$ :

$$
P[(\text { fnw,mse }) \mid(\mathrm{f}, \mathrm{m})]=\frac{P[(\mathrm{fnw}, \mathrm{mse})]}{P[(\mathrm{f}, \mathrm{m})]}=\frac{n_{\mathrm{fnw}} n_{\mathrm{mse}}}{n_{\mathrm{m}} n_{\mathrm{f}}} .
$$

Encounters between rats from the same birth locations are conditionalized on birth either in the southeast side or in the northwest side (se or nw), but not both:

$$
\begin{aligned}
& P[(\text { se }, \text { se }) \mid(\text { se }, \text { se }) \text { or }(\mathrm{nw}, \mathrm{nw})] \\
& =\frac{P[(\mathrm{se}, \mathrm{se})]}{P[(\mathrm{se}, \mathrm{se})]+P[(\mathrm{nw}, \mathrm{nw})]} \\
& =\frac{n_{\mathrm{se}}\left(n_{\mathrm{se}}-1\right)}{n_{\mathrm{se}}\left(n_{\mathrm{se}}-1\right)+n_{\mathrm{nw}}\left(n_{\mathrm{nw}}-1\right)} .
\end{aligned}
$$

\begin{tabular}{|c|c|c|c|c|}
\hline Conditional Prob. & Frequency & Expected & Observed & Statistics \\
\hline \multicolumn{5}{|l|}{ Sex } \\
\hline$P[(\mathrm{~m}, \mathrm{~m})]$ & .306 & 26.04 & 53 & $d f=2$ \\
\hline$P[(\mathrm{f}, \mathrm{m})]$ & .498 & 42.30 & 12 & $\chi^{2}=50.384$ \\
\hline$P[(\mathrm{f}, \mathrm{f})]$ & .196 & 16.70 & 20 & $p \ll .00001$ \\
\hline \multicolumn{5}{|l|}{ Male-male } \\
\hline$P[(\mathrm{mse}, \mathrm{mse})\}(\mathrm{m}, \mathrm{m})]$ & .447 & $23.71(23.9)$ & 30 & $d f=2$ \\
\hline$P[(\mathrm{mse}, \mathrm{mnw}) \mid(\mathrm{m}, \mathrm{m})]$ & .447 & $23.71(23.4)$ & 21 & $x^{2}=4.274$ \\
\hline$P[(\mathrm{mnw}, \mathrm{nw}) \mid(\mathrm{m}, \mathrm{m})]$ & .105 & $5.58(5.7)$ & 2 & $p>.11$ \\
\hline \multicolumn{5}{|l|}{ Female-female } \\
\hline$P[(\mathrm{fse}, \mathrm{fse}) \mid(\mathrm{f}, \mathrm{f})]$ & .307 & $6.13(6.2)$ & 4 & $d f=2$ \\
\hline$P[(\mathrm{fse}, \mathrm{fnw}) \mid(\mathrm{f}, \mathrm{f})]$ & .502 & $1.03(10)$ & 15 & $\chi^{2}=5.297$ \\
\hline$P[(\mathrm{fnw}, \mathrm{fnw}) \mid(\mathrm{f}, \mathrm{f})]$ & .192 & $3.84(3.9)$ & 1 & $p>.07$ \\
\hline \multicolumn{5}{|l|}{ Female-male } \\
\hline$P[(\mathrm{fse}, \mathrm{mse}) \mid(\mathrm{f}, \mathrm{m})]$ & .374 & $4.49(4.5)$ & 8 & $d f=3$ \\
\hline$P[(\mathrm{fnw}, \mathrm{mse}) \mid(\mathrm{f}, \mathrm{m})]$ & .297 & $3.56(3.6)$ & 1 & $\chi^{2}=4.93$ \\
\hline$P[(\mathrm{fse}, \mathrm{mnw}) \mid(\mathrm{f}, \mathrm{m})]$ & .183 & $2.20(2.2)$ & 2 & $p>.17$ \\
\hline$P[(\mathrm{fnw}, \mathrm{mnw}) \mid(\mathrm{f}, \mathrm{m})]$ & .146 & $1.75(1.7)$ & 1 & \\
\hline \multicolumn{5}{|l|}{ Birth location } \\
\hline$P[(\mathrm{se}, \mathrm{se}) \mid(\mathrm{se}, \mathrm{se})$ or $(\mathrm{nw}, \mathrm{nw})]$ & .729 & $33.54(34.6)$ & 42 & $d f=1$ \\
\hline$P[(\mathrm{nw}, \mathrm{nw})\}(\mathrm{se}, \mathrm{se})$ or $(\mathrm{nw}, \mathrm{nw})]$ & .271 & $12.46(11.3)$ & 4 & $\begin{array}{l}\chi^{2}=7.88 \\
p \leq .005 \\
(.01<p<.02)\end{array}$ \\
\hline \multicolumn{5}{|l|}{ Winner } \\
\hline$P[($ Win $(\mathrm{se})]$ & .500 & $19.5(18.9)$ & 32 & $d f=1$ \\
\hline$P[($ Win(nw) $]$ & .500 & $19.5(20.3)$ & 7 & $\begin{array}{l}\chi^{2}=16.026 \\
p<.00006 \\
(p \ll .001)\end{array}$ \\
\hline
\end{tabular}

The probabilities for sex and birth location encounters are listed in Table 3. In parentheses (under the "Expected"

Table 3

Reanalysis of Calhoun's (1962) Data on Agonistic Encounters From Table 42

Note-Numbers in parentheses are from Calhoun (1962), p. 197. 
column) are the values Calhoun (1962) reported using his approach, along with his original $p$ values. There are small differences owing to the error of inadvertently including self-agonistic encounters (and perhaps owing to round-off errors as well).

Finally, on this approach, it is clear that there are exactly $n_{\text {se }} n_{\text {nw }}$ possible agonistic encounters between southeastside and northwest-side rats. The expectation is that half of these encounters should be won by rats from the southeast side and half by rats from the northwest side-that is, $P[\mathrm{Win}(\mathrm{nw})]=P[\mathrm{Win}(\mathrm{se})]=0.5$. This is independent of the number of rats from either side. Calhoun (1962) did not come to the same conclusion (Table 3 ). This error appears to result from distinguishing 12 instead of 10 types of dyads and treating the problem as a sampling problem, rather than as a social network problem of dyadic interactions (compare Table 3 with Table 42, p. 197, of Calhoun, 1962). However, this error did not qualitatively affect Calhoun's conclusion that southeastborn rats are much more likely to win agonistic encounters (Table 3).

\section{GENERAL DISCUSSION}

The general approach developed in this paper appears to be broadly applicable to the analysis of complex dyadic interactions among animals. More specifically, the sex contact biases among pups reported here are consistent with the data reported by Deni et al. (1981), when corrected for the expected frequency of opposite-sex and same-sex contact (Table 1). The present results are also consistent with the view that huddling by pups primarily serves thermoregulation and energy conservation functions, because the observed deviations from expected frequencies of contact were small, only $3 \%$.

The patterns of sex contact biases observed among pups were qualitatively predictive of the male and female agonistic encounters observed by Calhoun (1962; see Table 3 under the "Sex" heading). Male-male agonistic encounters were higher than expected (potentially related to the lower than expected contact between male pups), male-female agonistic encounters were lower than expected (potentially related to the higher than expected contact between male and female pups), and femalefemale agonistic encounters were about what would be expected by chance (as was the frequency of contact between female pups). Thus, early sex preference or avoidances may have implications for the patterns of agonistic interactions observed in adulthood.

Prepubertal sex preferences may also have broad implications for the development of sexual attraction in other species, including humans (McClintock \& Herdt, 1996). Thus, these deviations from expected contact warrant further consideration. The first study, however, only indicates that there are sex biases in contact, and not which sexual preferences developmentally emerged or their magnitude. Further experiments are required to determine the causes of the higher than expected frequency of oppositesex contacts and the lower than expected frequency of male-male contacts.

\section{REFERENCES}

ALBERTS, J. (1978a). Huddling by rat pups: Group behavioral mechanisms of temperature regulation and energy conservation. Journal of Comparative \& Physiological Psychology, 92, 231-245.

ALBERTS, J. (1978b). Huddling by rat pups: Multisensory control of contact behavior. Journal of Comparative \& Physiological Psychology, 92, 220-230.

Alberts, J. R., \& Brunjes, P. C. (1978). Ontogeny of thermal and olfactory determinants of huddling in the rat. Journal of Comparative \& Physiological Psychology, 92, 897-906.

BARNETT, S. A. (1963). A study of behavior. London: Methuen.

CALHoun, J. B. (1962). The ecology and sociology of the Norway rat. Bethesda, MD: U.S. Department of Health, Education, and Welfare.

Deni, R., Martin, R., Makin, J. W., Longo, J., \& Andres, S. (1981). Sex preference and body orientation preference in huddling behavior of rat pups. Animal Learning \& Behavior, 9, 368-370.

MCClintock, M. K., \& Herdt, G. (1996). Rethinking puberty: The development of sexual attraction. Current Directions in Psychological Science, 5, 178-183.

Schank, J. C., \& AlberTs, J. R. (1997). Self-organized huddles of rat pups modeled by simple rules of individual behavior. Journal of Theoretical Biology, 189, 11-25.

Stollnitz, F. (1982). No sex preference and questionable bodyorientation preference in huddling behavior of rat pups. Animal Learning \& Behavior, 10, 269.

(Manuscript received January 24, 2000; revision accepted for publication July 5,2000 .) 